\title{
Quantum dot-photonic crystal chips for quantum information processing
}

\author{
Andrei Faraon, Dirk Englund, Ilya Fushman, Vanessa Sih, Jelena Vučković* \\ E.L. Ginzton Laboratory, Stanford University, Stanford, CA, 94305, USA
}

\begin{abstract}
We have recently developed a technique for local, reversible tuning of individual quantum dots on a photonic crystal chip by up to $1.8 \mathrm{~nm}$, which overcomes the problem of large quantum dot inhomogeneous broadening usually considered the main obstacle in employing such platform in practical quantum information processing systems. We have then used this technique to tune single quantum dots into strong coupling with a photonic crystal cavity, and observed strong coupling both in photoluminescence and in resonant light scattering from the system, as needed for several proposals for scalable quantum information networks and quantum computation.
\end{abstract}

Keywords: quantum information, photonic crystal, quantum dot

\section{INTRODUCTION}

Cavity quantum electrodynamics (CQED) is currently one of the most promising approaches for implementing quantum networks for information processing. ${ }^{1-4}$ Envisioned networks consist of elementary quantum systems, most often atom, ions, or quantum dots (QDs), interconnected by photonic channels. To enhance the interaction with light, these quantum systems are placed inside optical cavities. Simple quantum networks have already been proved using atoms coupled to macroscopic optical resonators. ${ }^{4,5}$ However, in order to implement a robust quantum network, it is generally accepted that it should be done with solid state systems. The solid state approach is to use QDs coupled to nanoresonators. Our group uses InAs QDs coupled to GaAs photonic crystal (PC) cavities. The advantage of using PCs is the scalability of this platform intensely investigated for on chip optical signal processing. The InAs QDs are good candidates for implementing quantum networks because of the possibility to manipulate their quantum states into a configuration suitable for quantum information storage. ${ }^{6}$

The main challenges regarding the construction of this type of solid state quantum networks is the difficulty to control the QDs. The InAs self-assembled QDs naturally grow randomly on the surface of the wafer, thus making the integration with nanoresonnators a non-trivial task. The deterministic positioning of a PC cavity on top of a QD has already been shown. ${ }^{7}$ However this does not represent a scalable solution because the difficulty of aligning many cavities in a quantum network increases exponentially with the number of quantum nodes. The most appealing solution would be for the QDs to be grown in a predefined array with precise positioning. Efforts are currently being done for mastering this technique.

Due to the randomness in the growth process, the spectrum of QDs exhibit inhomogeneous broadening. To create a quantum network, the QDs must be spectrally aligned with each other and with the nanoresonators they are embedded in. To overcome this difficulty, our group has developed two techniques ${ }^{8,9}$ that allow us to independently control the resonances of PC cavities and the QDs. One technique involves local control of the temperature on the chip. While this technique mainly addresses the tuning of QDs, we have developed a second technique that addresses the tuning of $\mathrm{PC}$ cavities. This technique is also local and can be used to independently tune PC cavities located on the same chip. Local tuning of $\mathrm{PC}$ cavities is part of our theoretical and experimental effort in controlling the properties of the PC devices. Beside the local tuning, our effort includes the design and fabrication of PC cavities with high quality factors and their integration with PC waveguides into an on-chip network. ${ }^{10-12}$

In the past few years we have made important steps toward the realization of on-chip quantum networks. We showed that the spontaneous emission rate of QDs can be enhanced by placing them in PC resonators. ${ }^{13}$

*To whom correspondence should be addressed. Email: jela@@stanford.edu 
This way the QD acts as a source of single photons that can be transfered on the chip via a PC waveguide. ${ }^{14}$ With the improvement of the quality of our photnic crystals we were able to achieve the strong coupling regime. One of our recent experiments integrates our latest developments in PC design and on-chip QD control to show coherent probing of a strongly coupled QD and giant optical nonlinearities.

All experiments presented in this paper are based on planar PC devices in GaAs with incorporated InAs QDs. QD wafers were grown by molecular beam epitaxy by our collaborators. They generally contained an active region consisting of a 150-160nm thick GaAs layer with a centered InAs/GaAs QD layer. The active layer was grown on an AlGaAs sacrificial layer (with high Al content), which is in turn grown on a GaAs substrate. The suspended PC structures were fabricated on such a wafer by a combination of electron beam lithography and dry etching (which creates a PC pattern in the top GaAs layer containing QDs), and a final wet etching step which dissolves a sacrificial layer underneath $\mathrm{PC}$ components.

The paper is organized as follows: in section 2 we discuss a simple quantum network that consists of a waveguide coupled single photon source. In section 3 we present our local tuning techniques used to independently tune the resonances of QDs and PC cavities. Section 4 is dedicated to our recent results on coherent probing of strongly coupled QDs ans observation of giant optical nonlinearities.

\section{GENERATION AND TRANSFER OF SINGLE PHOTONS ON A PHOTONIC CRYSTAL CHIP}

Sources of indistinguishable single photons are essential elements of quantum information processing systems required in quantum teleportation, ${ }^{15}$ linear-optics quantum computation, ${ }^{16}$ and several schemes for quantum cryptography. ${ }^{17}$ One way to implement single photon sources is to use a bulk semiconductor QD, but the efficiency of such a system is poor because most of the photons are lost in the substrate. Cavity quantum electrodynamics can be used ${ }^{18}$ to increase the photon extraction efficiency and reduce the duration of singlephoton pulses emitted from semiconductor systems. We have demonstrated cavity-enhanced single photon sources based on both microposts ${ }^{18,19}$ and PC cavities. ${ }^{13}$

An important component of quantum networks is a quantum channel for efficient information transfer between spatially separated nodes. ${ }^{3}$ For this reason, we have been pursuing single photon sources where the PC cavity output is redirected into a waveguide for efficient collection. In the rest of this section, we will describe single photon generation in such a structure, where emitted photons are outcoupled into the waveguide, and then transferred into another cavity on the chip (Fig. 1).

In Fig.1, a cavity-coupled QD single photon source is connected through a $25 \mu \mathrm{m}$ channel to an otherwise identical target cavity so that different cavities may be interrogated and manipulated independently. These end cavities with quality factor $Q \sim 1000$ have a near-minimum mode volume $V_{\text {mode }} \approx 0.74(\lambda / n)^{3}$ and allow a large spontaneous emission rate enhancement. This system provides a source of single photons with a high degree of indistinguishability (mean wavepacket overlap of $\sim 67 \%$ ), 12-fold spontaneous emission rate enhancement, spontaneous emission coupling factor $\beta \sim 0.98$ into the cavity mode, and high-efficiency coupling into a waveguide. These photons are transferred into the target cavity with a target/source field intensity ratio of $0.12 \pm 0.01$ (up to 0.49 observed in structures without coupled QDs), showing the system's potential as a fundamental component of a scalable quantum network for building on-chip quantum information processing devices.

A single-exciton transition is coupled to the source cavity (S) at $897 \mathrm{~nm}$ (see Fig. 1). The transition is driven resonantly through a higher-order excited QD state with a $878 \mathrm{~nm}$ pump from a Ti-Saph laser. The spontaneous emission rate enhancement is measured from the modified emitter lifetime, which is dominated by radiative recombination. ${ }^{13}$ A direct streak camera measurement puts the modified lifetime at 116ps (Fig. 2(b)). Compared to the average lifetime of $1.4 \mathrm{~ns}$ for QDs in the bulk semiconductor of this wafer, this corresponds to a Purcell enhancement of $F=12$. We estimate that this value of $F$ is about 13 times lower than for an ideally aligned dot, indicating spatial mismatch to $28 \%$ of the field maximum. The spontaneous emission coupling factor into the cavity mode is then $\beta=F /(F+f) \sim 0.98$, where $f \sim 0.2$ reflects the averaged spontaneous emission rate suppression into other modes due to the bandgap of the surrounding PC. ${ }^{13}$

We characterized the exciton emission by measurements of the second-order coherence and indistinguishability of consecutive photons. The second-order coherence $g^{(2)}\left(t^{\prime}\right)$ is measured with a Hanbury-Brown and Twiss 

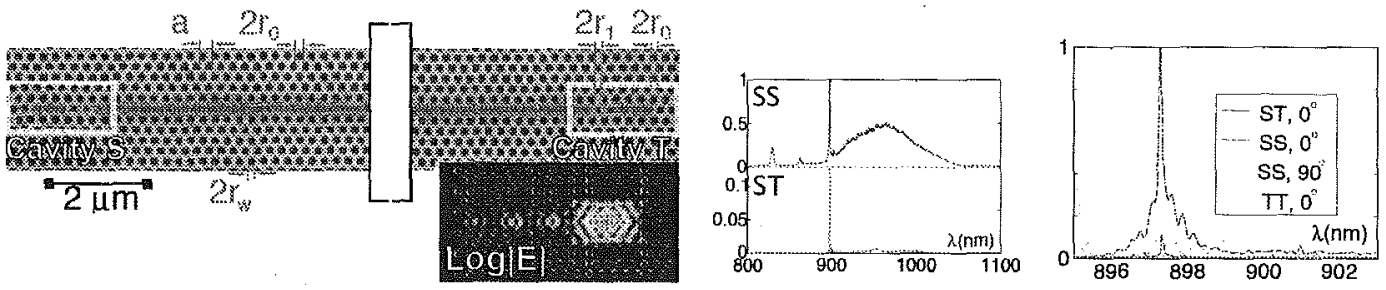

Figure 1. Coupled cavities system. Left: The identical source (S) and target (T) cavities are connected via the $25 \mu \mathrm{m}$ waveguide. Design parameters for the PC circuit are: $a=256 \mathrm{~nm}, r_{0}=0.3 a r_{1}=0.25 a, r_{0}=0.3 a$, and $r_{w}=0.25 a$. Electric field pattern is shown in the inset. Middle: Broad emission in cavity S (plot 'SS') is filtered into the target cavity (plot ' $\mathrm{ST}$ '), indicating matched cavity resonances with $\mathrm{Q}=1000$. Right: When the $\mathrm{QD}$ exciton at $897.3 \mathrm{~nm}$ in cavity $\mathrm{S}$ is pumped (resonantly at $878 \mathrm{~nm}$, with $460 \mu \mathrm{W}$ and $1 \mu \mathrm{m}$ focal spot pump), the emission is observed from S ('SS') and T ('ST'). The cross-polarized spectrum from $\mathrm{S}$ shows nearly complete quenching of QD emission ('SS, $90^{\circ}$ '). The line at $897.3 \mathrm{~nm}$ is only observed when $\mathrm{S}$ is pumped.

interferometer. ${ }^{13}$ When the QD in cavity $\mathrm{S}$ is pumped resonantly the photons observed from $\mathrm{S}$ show clear antibunching (Fig.2(a)), with $g^{2}(0)=0.35 \pm 0.01$.

Because of the shortened lifetime of the cavity-coupled QD exciton, the coherence time of emitted photons becomes dominated by radiative effects and results in high photon indistinguishability. ${ }^{20}$ We measured the indistinguishability using a Hong-Ou-Mandel (HOM) type setup. ${ }^{21}$ The QD is excited twice every $13 \mathrm{~ns}$, with a 2.3ns separation. The emitted photons are directed through a Michelson interferometer with a 2.3ns time difference. The two outputs are collected with single photon counters to obtain the photon correlation histogram shown in the inset of Fig. 2(b). The five peaks around delay $\tau=0$ correspond to the different possible coincidences on the beamsplitter of the leading and trailing photons after passing through the long or short arms $\mathcal{L}$ or $\mathcal{S}$ of the interferometer. If the two photons collide and are identical, then the bosonic symmetry of the state predicts that they must exit in the same port. This photon bunching manifests itself as antibunching in a correlation measurement on the two ports. This signature of photon indistinguishability is apparent in Fig. 2(b) in the reduced peaks near zero time delay. Following the analysis of Ref.21, the data (inset Fig.2(b)) indicate a mean wavefunction overlap of $I=0.67 \pm 0.18$, where we adjusted for the imperfect visibility (88\%) of our setup and subtracted dark counts in the calculation. Even with higher spontaneous emission rate enhancement, we expect that $I<0.80$ for resonantly excited $\mathrm{QDs}^{22}$ because of the finite relaxation time, measured here at 23ps by the streak camera.

We will now consider the transfer of single photons to the target cavity T. Experimentally, we verified photon transfer from $\mathrm{S}$ to $\mathrm{T}$ by spectral measurements (see Fig. 1): the exciton line is observed from $\mathrm{T}$ only if $\mathrm{S}$ is pumped. It is not visible if the waveguide or cavity $\mathrm{T}$ itself are pumped, indicating that this line originates from the QD coupled to cavity $\mathrm{S}$ and that a fraction of the emission is transferred to $\mathrm{T}$. This emission has the same polarization and temperature-tuned wavelength dependence as emission from S. Photon autocorrelation measurements on the signal from $\mathrm{T}$ indicate the antibunching characteristic of a single emitter when $\mathrm{S}$ is pumped (Fig. 2(c)). The signal-to-noise ratio is rather low because autocorrelation count rates are $\sim 0.014$ times lower than for collection from S. Nevertheless, the observed antibunching does appear higher, in large part because the background emission from cavity $\mathrm{S}$ is additionally filtered in the transfer to $\mathrm{T}$, as shown in Fig.1(b). Indeed, this filtering through the waveguide/cavity system suffices to bypass the spectrometer in the HBT setup (a 10nm bandpass filter was used to eliminate room lights). The count rate is about three times higher while antibunching, $g^{2}(0)=0.50 \pm 0.11$, is still clearly evident Fig. 2(d)). The largest contribution to $g^{2}(0)$ comes from imperfectly filtered photoluminescence near the QD distribution peak seen in Fig. 1(c). This on-chip filtering will be essential in future quantum information processing applications and should also find uses in optical communications as a set of cascaded drop filters.

To conclude this section, the experiment shows that single photons can be transferred on the chip which represents a fundamental step toward implementation of $\mathrm{PC}$ networks operating at the single photon level. 

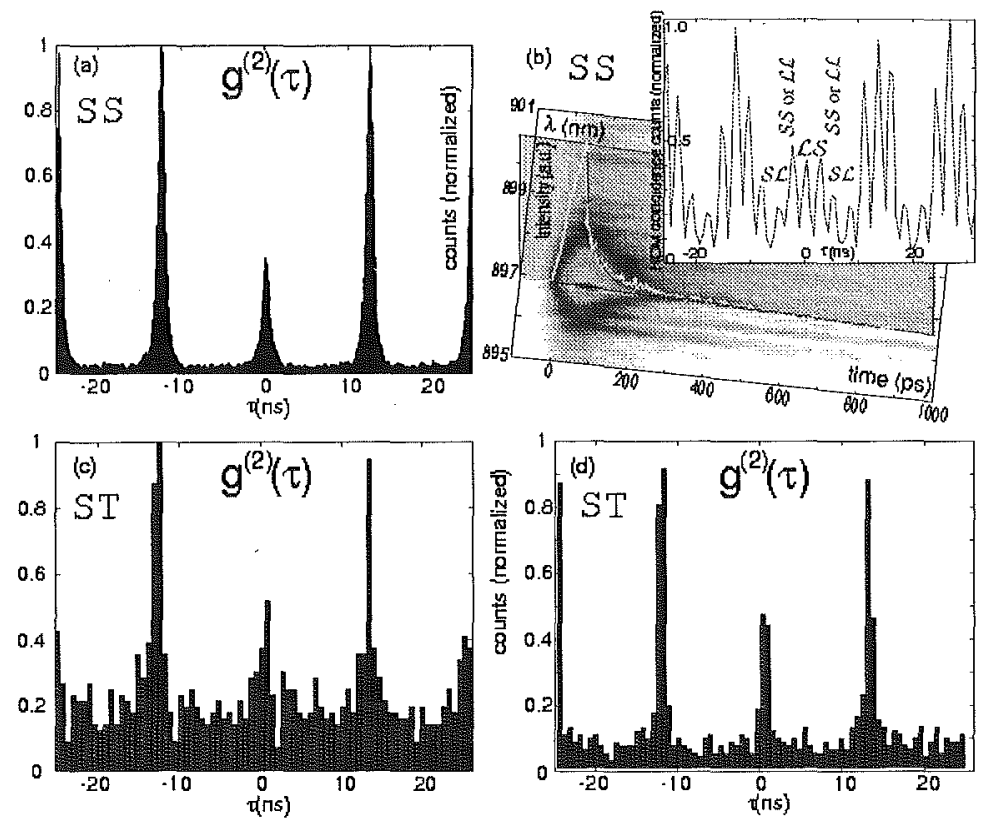

Figure 2. Single photon source characterization. (a) Autocorrelation data when cavity S pumped and collected. (b) Streak camera data indicate exciton lifetime $\tau=116 \mathrm{ps}$. The rise-time is measured at 23ps with a lower-density grating with higher time response (data not shown). Inset: Two-photon interference experiment. Colliding indistinguishable photons interfere, resulting in a decreased area of peak $\mathcal{L S}$. The area does not vanish largely because of non-zero $g^{(2)}(0)$ of the source. (c) Autocorrelation data when cavity S pumped and T is collected (with grating filter). (d) Cavity S pumped and $\mathrm{T}$ collected directly (no grating filter).

\section{LOCAL TUNING TECHNIQUES FOR QUANTUM DOTS AND PHOTONIC CRYSTAL CAVITIES}

In the previous section we show a component of quantum network with a single coupled QD. In order to implement a true network, more QDs coupled to cavities need to be interconnected. A major problem in realizing this task is spatial and spectral matching of distinct inhomogeneously broadened QDs. Spatial alignment can be achieved either by positioning the $\mathrm{PC}$ cavity on an already identified $\mathrm{QD},{ }^{7,23}$ or by relying on chance. For spectral alignment there are a few techniques that can be used to modify the emission wavelength of InAs QDs: Stark shift, ${ }^{24}$ Zeeman shift, ${ }^{25}$ temperature tuning ${ }^{26}$ and strain tuning. ${ }^{27}$ In this section we describe a technique ${ }^{8}$ for independent control of QDs, employing structures with high- $Q$ cavities whose temperature is controlled by laser beams. Our in-situ technique allows extremely precise spectral tuning of InAs QDs by up to $1.8 \mathrm{~nm}$ and of cavities of up to $0.4 \mathrm{~nm}$ (4 cavity linewidths). The technique is crucial for spectrally aligning distinct QDs on a PC chip and forms an essential step toward creating on-chip quantum information processing devices.

To achieve independent on-chip tuning, distinct regions containing the QDs of interest must be kept at different temperatures. Since GaAs is a good thermal conductor, on-chip local thermal insulation must be provided to achieve significant local heating. For this reason, we fabricated suspended PC structures with minimal thermal contact to the rest of the chip as shown in Fig.3. The fabricated structures (12 $\mu \mathrm{m}$ long, 4 $\mu m$ wide, $150 \mathrm{~nm}$ thick) consist of a PC cavity and a heating pad (Fig.3). To provide the thermal insulation needed for efficient device heating, the structure was connected to the rest of the chip by only six narrow bridges. The thermal conductivity of narrow $(\approx 100 \mathrm{~nm})$, cold $(4 \mathrm{~K}-10 \mathrm{~K}) \mathrm{GaAs}$ bridges is reduced by up to four orders of magnitude with respect to the bulk GaAs, ${ }^{28}$ thus improving the thermal insulation. We tested two devices with connection bridges of the same length $(2 \mu \mathrm{m})$ but different widths: $w=320 \mathrm{~nm}$ and $w=800 \mathrm{~nm}$. The temperature of the device was controlled by using a focused laser beam to heat up the pad next to the PC cavity. To minimize background photoluminescence in single QD measurements, the heating laser is tuned below the QD absorption frequency. A metal layer $(20 \mathrm{~nm} \mathrm{Cr} / 15 \mathrm{~nm} \mathrm{Au}$ ) was deposited on the heating pad to increase 


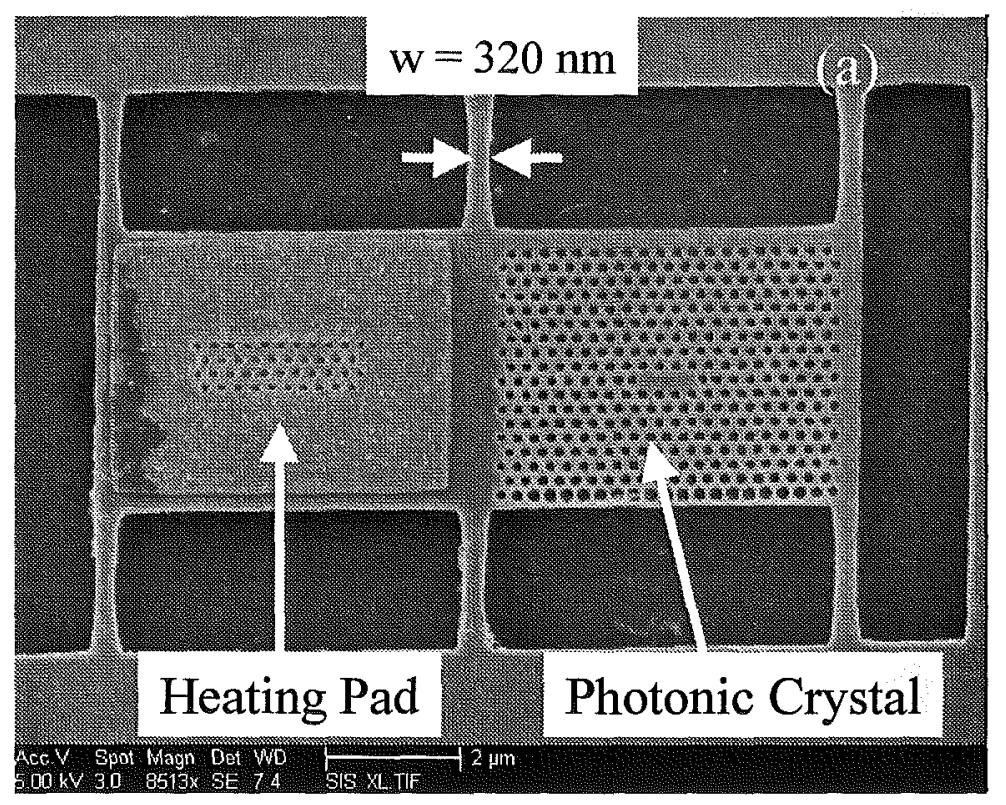

Figure 3. Scanning electron microscope image of the fabricated structure showing the PC cavity, the heating pad and the connection bridges. The temperature of the structure was controlled with a laser beam (960 $\mathrm{nm})$ focused on the heating pad.

heat absorption. Assuming that $10^{-2} \mathrm{~mW}$ of heat is absorbed in the heating pad, we expect the temperature of the membrane to increase by a few tens of Kelvins.

The local QD tuning measurements were performed in a continuous flow liquid helium cryostat maintained at 10K. A Ti:Saph laser tuned at $855 \mathrm{~nm}$ was used to excite the QDs while a $960 \mathrm{~nm}$ laser diode acted as the heating laser. Using a pinhole we collected photoluminescence from a QD located inside the PC slab. By increasing the power of the heating laser, QD emission was observed to redshift (Fig.4 (a)). The QD line-width broadens with increasing heating pump power, as expected from experiments where the full sample is heated. We were able to tune the QD by $1.4 \mathrm{~nm}$ while the linewidth broadened from $0.04 \mathrm{~nm}$ to $0.08 \mathrm{~nm}$. The QD could be further shifted by $1.8 \mathrm{~nm}$ but the PL intensity dropped rapidly. The measurement of the QD shift on the thickness of the connection bridges is shown in Fig.4 (c). The data in Fig.4 (d) shows the QD tuning by changing the temperature of the cryostat.

To show the compatibility of this local tuning technique with single photon measurements and quantum information processing, we proved anti-bunched single photon emission from an exciton transition using a HandburyBrown-Twiss interferometer while the emission line was shifted by $0.8 \mathrm{~nm}$ (Fig.4 (b)). Antibunching can be measured as long as the thermal energy is not larger than the confining energy of the $\mathrm{QD}$, which in our case corresponds to a detuning of $\approx 1.4 \mathrm{~nm}$.

Not only the QDs but also the PC cavities shift their resonant frequency with temperature. The local heating technique was used to shift a PC cavity located on the $w=320 \mathrm{~nm}$ structure. Using the same heating power as for the QD tuning, we observed the cavity resonance red shift by up to $0.48 \mathrm{~nm}$ (Fig.5 (a) and (b)), about 3 times less than the QD shift. The quality factor of the cavity dropped from 7600 to 4900 .

This proof of concept experiment shows the local tuning of PC cavities and QDs that are not integrated into a PC circuit. Implementing the local tuning technique in an integrated optical circuit is only a matter of thermal design. A prototype of such a device consisting of several PC cavities coupled to a common PC waveguide is shown in Fig.6.

As discussed above, the resonance of both the PC cavities and the QDs is affected by the change in temperature. In a real quantum network, one should have the ability to independently control the resonances of 

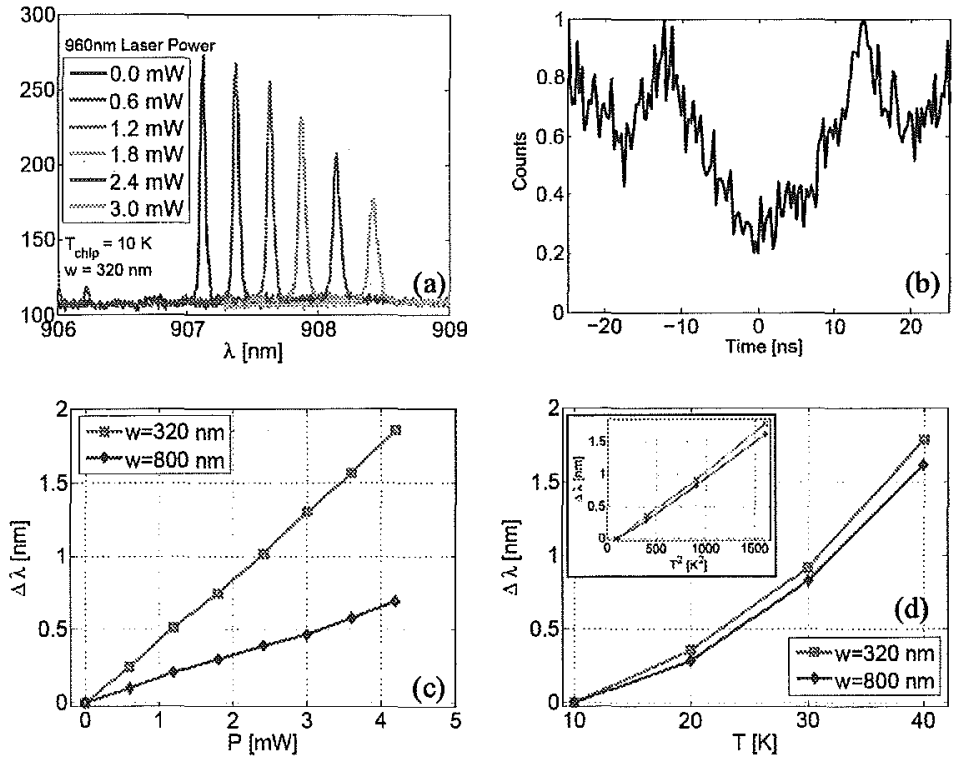

Figure 4. (a) Quantum dot tuning vs. heating pump power. The structure is connected to the substrate by bridges measuring $320 \mathrm{~nm}$ in width. The quantum dot emission shifts by $1.4 \mathrm{~nm}$ while increasing the heating laser power to 3 $\mathrm{mW}$. Only a small fraction of the heating laser power is absorbed in the metal pad. (b) Autocorrelation measurement showing single photon anti bunching while the QD was detuned by $0.8 \mathrm{~nm}$ using the local tuning technique. (c) Dependence of the QD detuning on the heating laser power. The two data sets correspond to structures with different thermal contact to the substrate (320 $\mathrm{nm}$ and $800 \mathrm{~nm}$ bridges). (d) QD temperature tuning by changing the temperature of the entire chip by heating the cryostat. The inset shows that the detuning is linear in $T^{2}$.
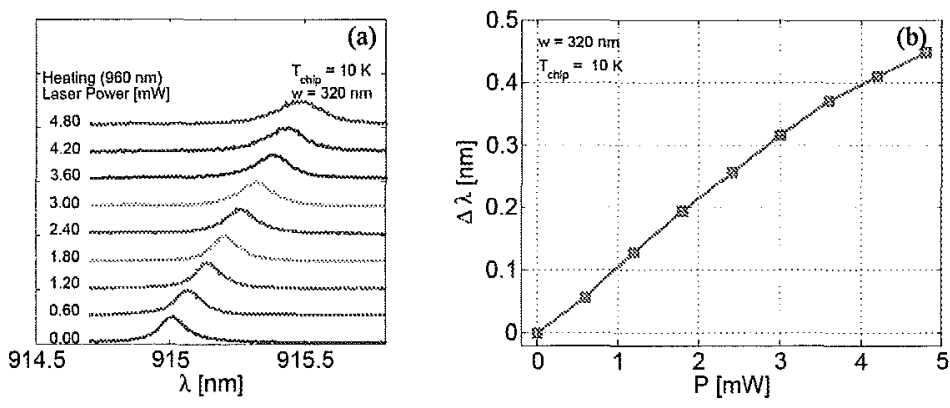

Figure 5. (a) Detuning of the PC cavity resonance with increasing temperature due to local heating. (b) Dependence of the $\mathrm{PC}$ cavity resonance wavelength on the local heating power. 


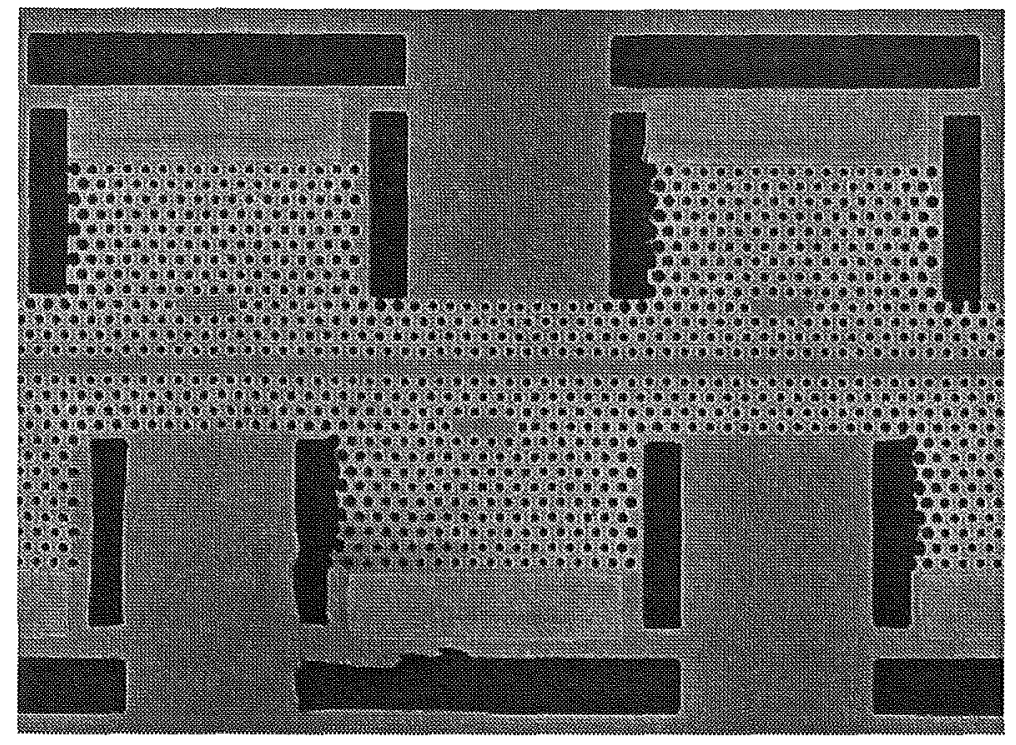

Figure 6. Integrated PC device. Several PC cavities are coupled to a common rail PC waveguide. Each cavity has a heating pad and is surrounded by trenches for thermal insulation. The resonance of each cavity can be independently controlled by an external heating laser beam.

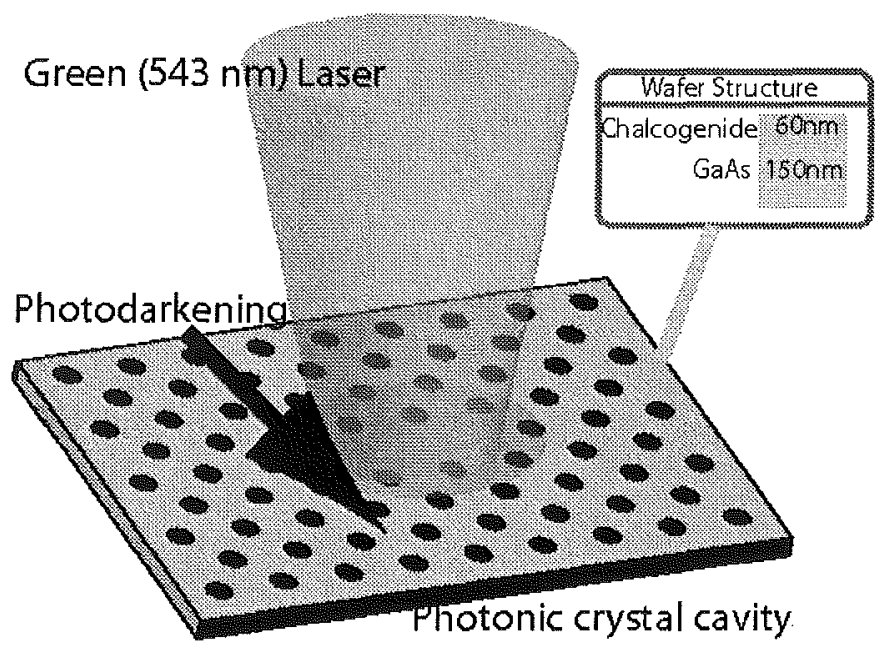

Figure 7. Schematic of the method for local cavity tuning. A layer of $\mathrm{As}_{2} \mathrm{~S}_{3}$ is deposited on top of the PC cavity. Then a laser tuned close to the $\mathrm{As}_{2} \mathrm{~S}_{3}$ band gap is focused on the cavity, increasing the effective refractive index and causing a resonance red-shift.

both QDs and nanoresonators. We are currently developing a method for tuning GaAs PC devices, based on chalcogenide glasses. ${ }^{9}$ Chalcogenide glasses quasi-permanently change their optical properties when illuminated with light above their band gap, and have been used to tune optical devices as quantum cascade lasers. ${ }^{29}$ The tuning of PCs devices directly fabricated in chalcogenide glasses has already been shown in Ref.30. Our approach is to deposit a chalcogenide glass layer on prefabricated GaAs/InAs devices. After deposition, an external laser beam can be used to locally change the refractive index on the PC cavity, as depicted in Fig.7. The local index change makes the cavity resonance to red-shift and thus can be used for spectral allignemnt on the chip. 


\section{COHERENT PROBING OF A STRONGLY COUPLED QUANTUM DOT IN A PHOTONIC CRYSTAL CAVITY}

Cavity quantum electrodynamics studies the physics of light emitters inside optical cavities. In the case of a singe emitter coupled to a resonator the relevant parameters are: the coupling rate $g$ between the dipole and the resonator field, the decay rate $\kappa$ of the field in the resonator, and the free space emission rate of the dipole $\gamma$. When $g$ is greater than $\kappa$ and $\gamma$, the system is strongly coupled. In this regime, in case the system is excited, the energy can coherently oscillate between the cavity mode and the dipole, thus giving rise to Rabi oscillations. For a QD resonant with the cavity, this effect can be observed as a degenerately split spectrum in photoluminescence or coherent light scattering. The first observations of strong coupling in nano-resonators have been done in photoluminescence. ${ }^{7,31}$ However, in order to use these system for quantum information science, ${ }^{3,32-36}$ the probing needs to be done in a coherent manner. We demonstrated coherent probing in Ref.37 where we showed that a QD can block the photons from entering the cavity when resonant with it.

For this experiment we used a similar structure as the one showed in Fig.3. A linear three-hole defect in the photonic crystal forms the cavity ${ }^{38}$ with a resonant mode at $\lambda=926 \mathrm{~nm}$ and measured measured quality factor $Q=1.0 \cdot 10^{4}\left(\Delta \lambda_{c a v}=0.10 \mathrm{~nm}\right)$. We observe a polariton splitting of $0.05 \mathrm{~nm}$.

A narrow-bandwidth laser was used to coherently scan the cavity resonance with a high resolution. To enhance the signal to noise ratio, we set up a cross-polarization measurement as explained in Fig.8(b). The horizontal $|H\rangle$ component of the scattered probe beam then carries the cavity reflectivity $R$ as given by Eq. 2 . To avoid difficulties related to laser stability and power normalization, we keep the laser wavelength fixed and instead scan the cavity and QD using the local temperature-tuning technique ${ }^{8}$ described in the section 3 . The sample is kept at $27 \mathrm{~K}$ and probed using a confocal microscope setup Fig.8(a). In Fig.8(d) we show the reflectivity signal from a cavity without coupled QDs. In this case the cavity resonance is swept through the laser beam by using local temperature tuning. To maximize the signal to noise ratio we used a half wave plate in front of the setup to compensate for non optimal orientation of the cavity (see Fig.8(a)).

The strong coupling regime was first confirmed by exciting the system with laser light tuned at 780nm above the band gap of GaAs as observing the photoluminescece. Anticrossing between the two polaritons is clearly observed in Fig.9(c), as the QD is tuned into resonance with the cavity.The splitting between the polaritons is described by

$$
\omega_{ \pm}=\frac{\omega_{c}+\omega_{d}}{2}-i \frac{\kappa+\gamma}{2} \pm \sqrt{g^{2}+\frac{1}{4}(\delta-i(\kappa-\gamma))^{2}}
$$

where $\omega_{c}$ denotes the cavity frequency, $\omega_{d}$ the QD frequency, $\delta=\omega_{d}-\omega_{c}$ the QD/cavity detuning, cavity field decay rate $\kappa / 2 \pi=16 \mathrm{GHz}$ (linewidth $0.1 \mathrm{~nm}$ ), Rabi frequency $g / 2 \pi=8 \mathrm{GHz}$ (from Rabi splitting of $2 g$ corresponding to $0.05 \mathrm{~nm}$ ), and the dipole decay rate without the cavity $\gamma / 2 \pi \approx 0.1 \mathrm{GHz}$. As $g \approx \kappa / 2$, the cavity/QD system operates at the onset of strong coupling, ${ }^{39}$ as was also the case for other QD-PC cavity QED experiments done in photoluminescence. ${ }^{7,31}$

Having verified the strong coupling, the reflectivity was probed by setting the probe laser at five different detuning from the anticrossing frequency. An excitation power less than $3 \mathrm{nW}$ was used in order to keep a low average photon number in the cavity. The spectrum in Fig.10 shows the reflectivity signal as a function of temperature scan for the case when the probe laser was detuned by $0.83 g$ from anticrossing. In this data set, the temperature tuning is used to sweep the QD and cavity back and forth through the probe laser. Instead of observing a Lorentzian-shaped cavity spectrum as in Fig.8(d), a drop in the reflected signal is observed at the QD wavelength which is the signature of coherent probing of the system.

The reflected signal from the described cavity is derived following Refs. 35 and 33 . The spectrum of the reflected probe signal after the polarizing beam splitter is then given by

$$
R=\eta\left|\frac{\kappa}{i\left(\omega_{c}-\omega\right)+\kappa+\frac{g^{2}}{i\left(\omega_{d}-\omega\right)+\gamma}}\right|^{2},
$$

where $\eta$ accounts for the efficiency of coupling to and collecting from the cavity. We fit this relation to the observed spectrum, using the system parameters and the tracked wavelength of the cavity and the QD Fig.10(A). 

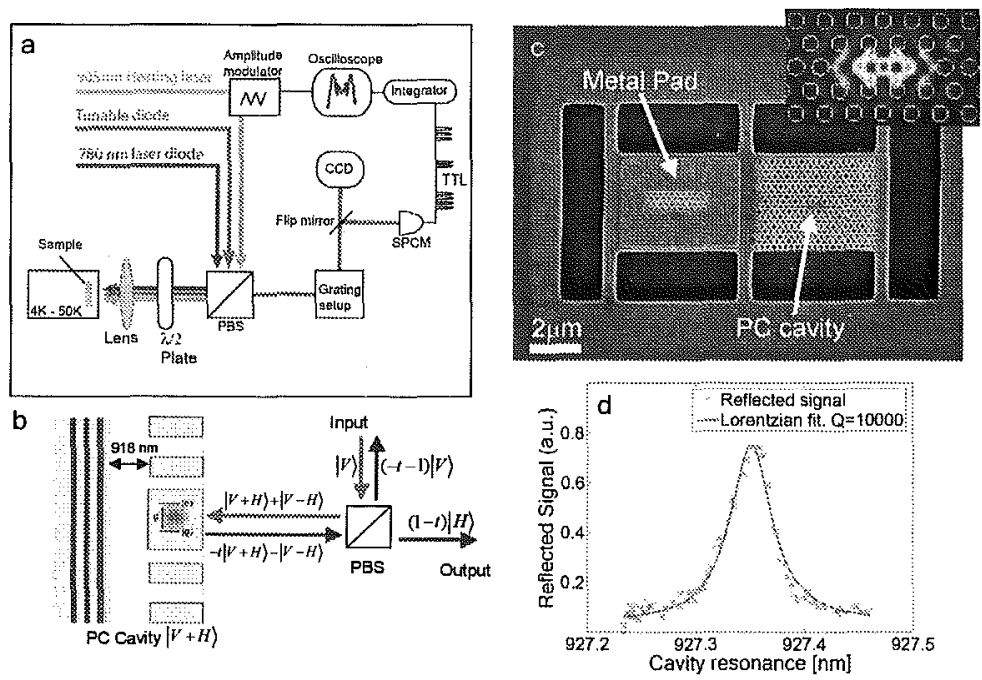

Figure 8. Experiment setup. (a) Confocal microscope setup. A $780 \mathrm{~nm}$ laser diode excites photoluminescence, while a $905 \mathrm{~nm}$ modulated Ti:Sapphire laser locally heats the sample to tune cavity and QD. ${ }^{8}$ The reflectivity is measured with a narrow-band tunable diode laser (focal spot diameter $\sim 1 \mu \mathrm{m}$ for all beams). A grating setup monitors the photoluminescence and filters the reflectivity signal from background noise. The filtered reflected signal is detected by a Single Photon Counting Module (SPCM). (b) Principle of the reflectivity measurement off a PC cavity. A vertical $\left(|V\rangle\right.$-polarized) probe laser is directed onto the linearly polarized cavity oriented at $45^{\circ}(|V+H\rangle)$. Due to interaction with the cavity, the $|H+V\rangle$ component of the probe beam is reflected with a frequency-dependent coefficient $-t(\omega)$. The $|V-H\rangle$ component reflects directly with a $\pi$ phase shift. The polarizing beam splitter (PBS) passes $|H\rangle$, giving a signal that is proportional to $|1-t|^{2}$ on the detector (see Eq.2). (c) Suspended structure composed of a heating pad and a PC cavity. The heating laser incident on the metal pad controls local temperature. ${ }^{8}$ Inset: simulated electric field intensity of PC cavity. (d) Reflectivity spectrum obtained by tuning an empty cavity (no coupled QD) through the probe laser, indicating $Q=1.0 \cdot 10^{4}$.

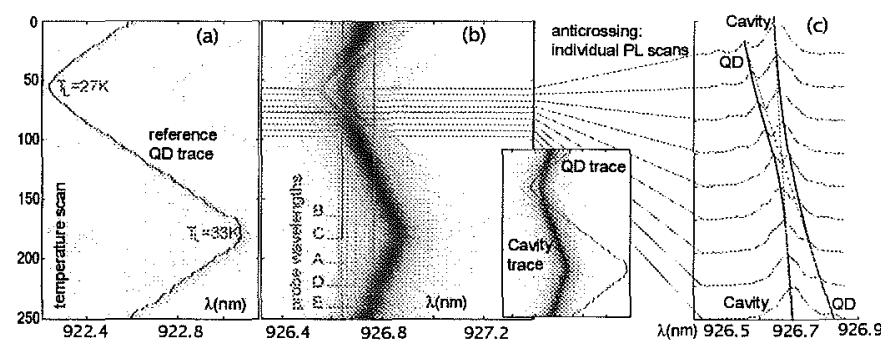

Figure 9. Photoluminescence of a single QD tuned through strong coupling to a PC cavity, excited using an above-band pump beam (780 nm wavelength, with $20 \mathrm{nW}$ power incident on the sample surface). Tuning of the QD through the cavity resonance is achieved following our earlier work, ${ }^{8}$ with a heating beam intensity-modulated between $6 \mu \mathrm{W}$ and $300 \mu \mathrm{W}$ to change local temperature $T_{L}$ from $27-33 \mathrm{~K}$. (a) A reference QD is used for tracing the wavelength of the strongly coupled QD, since dots that are closely spaced in wavelength exhibit identical temperature tuning behavior. The heating beam power is modulated with a triangular pattern and shifts the QD nearly linearly. (b) PL emission shows the strongly coupled $\mathrm{QD}$ tuned in and out of resonance with a PC cavity $\left(Q \approx 1.0 \cdot 10^{4}\right)$. In the reflectivity measurements, the aboveband pump is switched off and the cavity/QD system probed at different detunings of the reflected laser beam from the point of anticrossing (lines A-E). Inset: QD and cavity traces. (c) Individual PL cross-sections show anticrossing between QD and cavity, with measured Rabi splitting of $0.05 \mathrm{~nm}$ (corresponding to $2 g$, where the coupling strength $g / 2 \pi=8 \mathrm{GHz}$ ). As a guide the the eye, we show the wavelengths of the uncoupled QD and cavity (red line). 

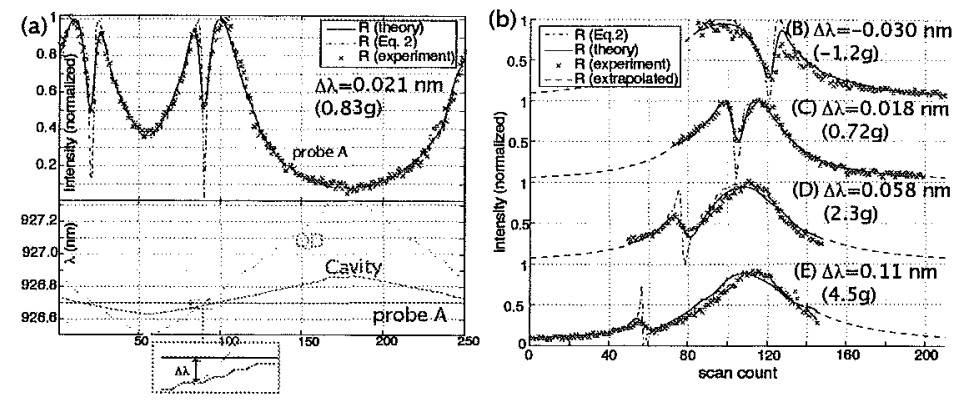

Figure 10. QD-controlled cavity reflectivity at different probe wavelengths A-E, as indicated in Fig.9(b). (a) Reflectivity spectrum of probe laser as function of QD and cavity detunings, as determined from corresponding PL spectra (see Fig.9). The probe laser is detuned by $\Delta \lambda=0.021 \mathrm{~nm}$ (corresponding to $\Delta \lambda=0.83 \mathrm{~g}$ ) from the anticrossing point between QD and cavity (see inset). Ideal theoretical plots are calculated from Eq.2. Also shown are theoretical plots that take into account a $\sim 0.005 \mathrm{~nm}$-jitter of cavity and QD wavelength resulting from the heating laser power fluctuation. (b) Probe laser at various detunings $\Delta \lambda$ from the anticrossing point samples different QD-cavity detunings. Incomplete scans result from the limited range of temperature tuning.

In doing the fit we also take into account temperature fluctuations that arise from instabilities in the power of the heating laser. In Fig.10(B-E) we show the reflectivity data for other probe wavelengths. Each spectrum (B-E) captures the QD-induced dip at different detunings from the anticrossin wavelength. These detunings range from $-1.2 g(-0.03 \mathrm{~nm})$ to $4.5 g(0.11 \mathrm{~nm})$. For each scan, the dip in reflectivity occurs precisely where the QD crosses the laser beam.

The depth of the dip in reflectivity depends on the input power of the probe laser. At high power the QD saturates, giving rise to a giant optical nonlinearity. This nonlinearity is explored in Fig.11. The input power $P_{i n}$ is increased from the low-excitation limit at $5 \mathrm{nW}$ before the objective (corresponding to average cavity photon number $\left\langle n_{\text {cav }}\right\rangle \approx 0.003$ in a cavity without QD) to the high-excitation regime with $P_{\text {in }} \approx 12 \mu \mathrm{W}$ (corresponding $\left\langle n_{\text {cav }}\right\rangle \approx 7.3$ ). Here, $\left\langle n_{\text {cav }}\right\rangle$ is estimated as $\eta P_{i n} / 2 \kappa \hbar \omega_{c}$, where $\eta \approx 1.8 \%$ is the coupling efficiency into the cavity at this wavelength. Fig.11(a) shows the QD-induced reflectivity dip vanishing as $P_{i n}$ is increased roughly by three orders of magnitude. The data is summarized in Fig.11(b), where we plot the reflectivity $R$ at the QD detuning $\Delta \lambda=-0.012 \mathrm{~nm}$, normalized by the reflectivity value $R_{0}$ for an empty cavity at the same wavelength as the probe laser (i.e., for $g \rightarrow 0$ ). Our results agree with the theoretical model (solid curve) and previous measurements in atomic systems. ${ }^{40}$ Saturation begins at $\sim 1 \mu \mathrm{W}$ of incident power (measured before the objective), corresponding to $\left\langle n_{\text {cav }}\right\rangle \approx 1 / 2$. Taking into account the coupling efficiency $\eta$, this implies a saturation power inside the cavity of only $\sim 20 \mathrm{nW}$, in agreement with previous predictions for giant optical nonlinearity in a microcavity. ${ }^{41}$

In conclusion, we have shown that a QD can be used to change the reflectivity spectrum of a photonic crystal cavity. This experiment proves coherent probing of solid state strongly coupled systems. This has far reaching implications for quantum information processing in solid-state systems, as it opens the door to high-fidelity controlled phase gates, ${ }^{33}$ single photon detection, ${ }^{42}$ coherent transfer of the QD state to photon state, ${ }^{3}$ and quantum repeaters employing nondestructive Bell measurements with the addition of a third long lived QD level. $^{35}$

\section{CONCLUSION}

In this paper we presented our latest experimental progress in the study of on-chip cavity quantum electrodynamics. We showed that single photons can be generated in a photonic crystal cavity and then transferred to another cavity via a photonic crystal waveguide. This simple system represents a vital component for future solid state quantum networks were QDs in photonic crystal cavities are connected via photonic crystal waveguides. To solve the problem of inhomogeneous broadening of QDs, we developed two local tuning techniques that allow us to independently tune QDs and photonic crystal cavities located on the same chip. One tuning method relies on local control of temperature. The other method, based on chalcogenide glasses, relies on local control of the 


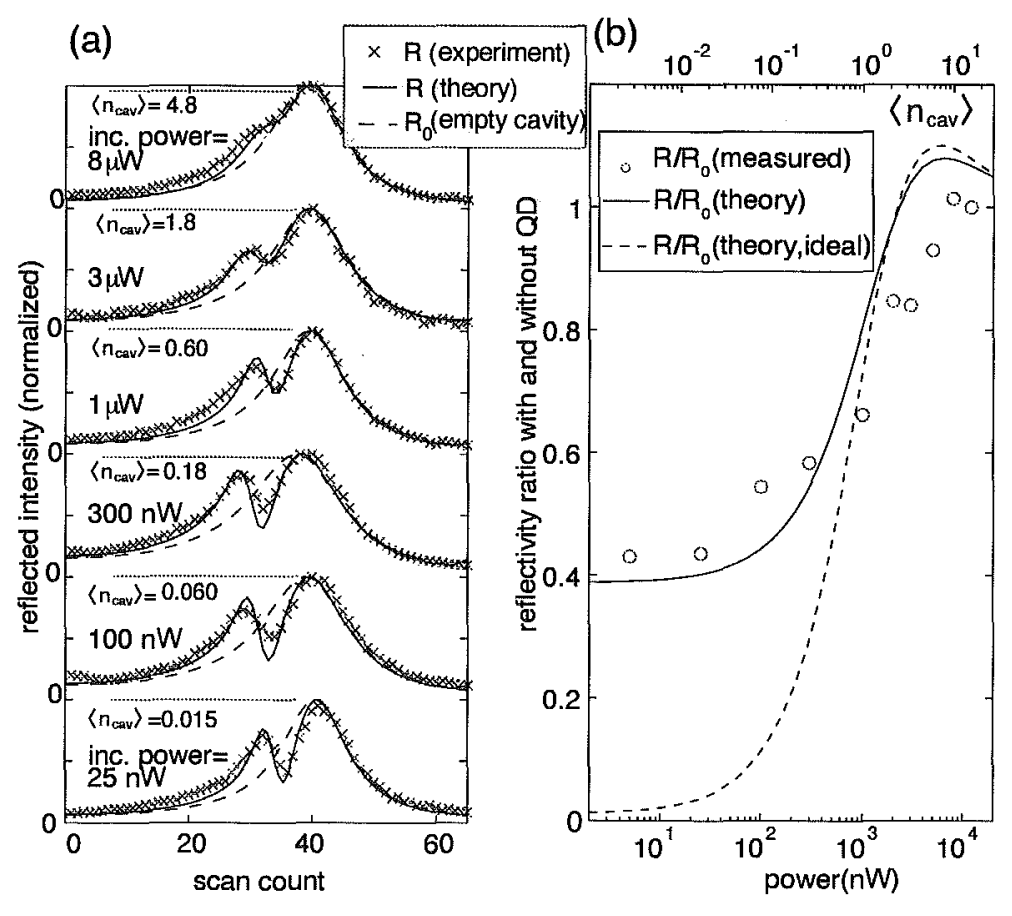

Figure 11. QD-controlled cavity reflectivity versus probe beam power for probe laser detuning of $\Delta \lambda=-0.012 \mathrm{~nm}$ from the anticrossing point. (a) Reflected intensity vs. incident beam power (measured before the objective) ranging from low-excitation to saturation regimes. The measured reflectivity is fit by a numerical solution to the full master equation. Solutions are convolved with a Gaussian filter with $\mathrm{FWHM}=0.005 \mathrm{~nm}$ to account for thermal fluctuations (solid curves). The scale for the calculated mean photon number $\left\langle n_{c a v}\right\rangle$ is also indicated. Also plotted is the expected reflectivity $R_{0}$ when the QD is removed (dashed curve). (b) Reflectivity at $\Delta \lambda=-0.012$, normalized by empty-cavity reflectivity at the same wavelength, as a function of probe laser power. Saturation begins near $1 \mu \mathrm{W}$ of input power, corresponding to $\left\langle n_{\text {cav }}\right\rangle \approx 1 / 2$. The dashed curve shows the reflectivity ratio if no thermal fluctuations were present. At large power, both curves tend to unity as the $\mathrm{QD} /$ cavity spectrum approaches the Lorentzian shape of the empty cavity. 
refractive index. The local temperature control has been succesfully used to realize coherent probing of strongly coupled QDs in photonic crystal cavities. The coherent probing is key to future experiments as coherent transfer of the QD state to photon state, quantum repetears or nonlinear optics at the single photon level via photon blockade effect.

\section{REFERENCES}

1. Daniel Loss and David P. DiVincenzo. Quantum computation with quantum dots. Physical Review A, 57(1):120-126, 1998.

2. A. Imamoglu, D. D. Awschalom, G. Burkard, D. P. DiVincenzo, D. Loss, M. Sherwin, and A. Small. Quantum Information Processing Using Quantum Dot Spins and Cavity QED. Physical Review Letters, 83(20):4204-4207, November 1999.

3. J. I. Cirac, P. Zoller, H. J. Kimble, and H. Mabuchi. Quantum State Transfer and Entanglement Distribution among Distant Nodes in a Quantum Network. Physical Review Letters, 78(16):3221-24, April 1997.

4. D. Felinto, C. W. Chou, J. Laurant, E. W. Schomburg, H. De Riedmatten, and H. J. Kimble. Conditional control of the quantum states of remote atomic memories for quantum networking. Nature Physics, 2:844$848,2006$.

5. D. L. Moehring, P. Maunz, S. Olmschenk, K. C. Younge, D. N. Matsukevich, L.-M. Duan, and C. Monroe. Entanglementof single-atomquantumbits at adistance. Nature, 449:68-72, 2007.

6. Mete Atature, Jan Dreiser, Antonio Badolato, Alexander Hogele, Khaled Karrai, and Atac Imamoglu. Quantum-Dot Spin-State Preparation with Near-Unity Fidelity. Science, 312:551-553, april 2006.

7. K. Hennessy, A. Badolato, M. Winger, D. Gerace, M. Atatüre, S. Gulde, S. Falt, E.L. Hu, and A. Imamoglu. Quantum nature of a strongly coupled single quantum dot-cavity system. Nature, 445:896-899, Feb 2007.

8. Andrei Faraon, Dirk Englund, Ilya Fushman, Jelena Vuckovic, Nick Stoltz, and Pierre Petroff. Local quantum dot tuning on photonic crystal chips. Applied Physics Letters, 90(213110), 2007.

9. Andrei Faraon, Dirk Englund, Douglas Bulla, Barry Luther-Davies, Benjamin J. Eggleton, Nick Stoltz, Pierre Petroff, and Jelena Vuckovic. Local tuning of photonic crystal cavities using chalcogenide glasses. ArXiv, arXiv:0711.0772v2, Nov 2007.

10. D. Englund, I. Fushman, and J. Vučković. General Recipe for Designing Photonic Crystal Cavities. Optics Express, 12(16):5961-75, August 2005.

11. D. Englund and J. Vučković. A direct analysis of photonic nanostructures. Optics Express, 14(8):3472-3483, 2006.

12. Andrei Faraon, Edo Waks, Dirk Englund, Ilya Fushman, and Jelena Vuckovic. Efficient photonic crystal cavity-waveguide couplers. Applied Physics Letters, 90(073102), 2007.

13. D. Englund, D. Fattal, E. Waks, G. Solomon, B. Zhang, T. Nakaoka, Y. Arakawa, Y. Yamamoto, and J. Vučković. Controlling the Spontaneous Emission Rate of Single Quantum Dots in a Two-Dimensional Photonic Crystal. Physical Review Letters, 95(013904), July 2005.

14. D. Englund, A. Faraon, B. Zhang, Y. Yamamoto, and J. Vučković. Generation and Transfer of Single Photons on a Photonic Crystal Chip. Optics Express, 15:5550-5558, 2007.

15. D. Bouwmeester, J. Pan, K. Mattle, M. Eibl, H Weinforuter, and A. Zeilinger. Experimental quantum teleportation. Nature, 390:575-9, 1997.

16. E. Knill, R. Laflamme, and G. J. Milburn. A scheme for efficient quantum computation with linear optics. Nature, 409:4652, 2001.

17. M. A. Nielsen and I. L. Chuang. Quantum Computation and Quantum Information. Cambridge Univ. Press, Cambridge, 2000.

18. J. Vuckovic, C. Santori, D. Fattal, M. Pelton, G. Solomon, and Y. Yamamoto. in Optical Microcavities, edited by K. Vahala. World Scientific, Singapore, 2004.

19. J. Vučković, D. Fattal, C. Santori, G. Solomon, and Y. Yamamoto. Enhanced single photon emission from a quantum dot in a micropost microcavity. Applied Physics Letters, 82:3596-3598, May 2003.

20. A. Kiraz, M. Atatüre, and I. Imamoğlu. Quantum-dot single-photon sources: Prospects for applications quantum-information processing. Phys. Rev. A, 69:p.032305-1-032305-10, March 2004. 
21. C. Santori, D. Fattal, J. Vučković, G. S. Solomon, and Y. Yamamoto. Indistinguishable photons from a single-photon device. Nature, 419(6907):594-7, October 2002.

22. J. Vučković, D. Englund, D. Fattal, and E. Waks Y. Yamamoto. Generation and manipulation of nonclassical light using photonic crystals. Physica E, 31(2), 2006.

23. A. Badolato, K. Hennessy, M. Atatüre, J. Dreiser, E. Hu, P.M. Petroff, and A. Imamoglu. Deterministic Coupling of Single Quantum Dots to Single Nanocavity Modes. Science, 308(5725):1158 - 1161, May 2005.

24. A. Högele, S. Seidl, M. Kroner, K. Karrai, R.J. Warburton, B.D. Gerardot, and P.M. Petroff. VoltageControlled Optics of a Quantum Dot. Physical Review Letters, 93(217401), Nov 2004.

25. D. Haft, C. Schulhauser, A.Q. Govorov, R.J. Warburton, K. Karrai, J.M. Garcia, W. Schoedfeld, and P.M. Petroff. Magneto-optical properties of ring-shaped self-assembled InGaAs quantum dots. Physica E, 13:165-169, 2002.

26. A. Kiraz, P. Michler, C. Becher, B. Gayral, A. Imamoglu, L. Zhang, E. Hu, W.V. Schoenfeld, and P.M. Petroff. Cavity-quantum electrodynamics using a single InAs quantum dot in a microdisk structure. Applied Physics letters, 78(25):3932-3934, June 2001.

27. Stefan Seidl, Martin Kroner amd Alexander Högele, Khaled Karrai, Richard J. Warburton, Antonio Badolato, and Pierre M. Petroff. Effect of uniaxial stress on excitons in a self-assembled quantum dot. Applied Physics letters, 88(203113), 2006.

28. W. Fon, K.C. Schwab, J.M. Worlock, and M.L. Roukes. Phonon scattering mechanisms in suspended nanostructures from 4 to 40 K. Physical Review B, 66(045302), 2002.

29. Shanshan Song, Scott S. Howard, Zhijun Liu, Afusat O. Dirisu, Claire F. Gmachl, and Craig B. Arnold. Mode tuning of quantum cascade lasers through optical processing of chalcogenide glass claddings. Applied Physics Letters, 89(041115), 2006.

30. Michael W. Lee, Christian Grillet, Cameron L.C. Smith, David J. Moss, Benjamin J. Eggleton, Darren Freeman, Barry Luther-Davies, Steve Madden, Andrei Rode, Yinlan Ruan, and Yong hee Lee. Photosensitive post tuning of chalcogenide photonic crystal waveguides. Optics Express, 15(3):1277, 2007.

31. T. Yoshie, A. Scherer, J. Hendrickson, G. Khitrova, H. M. Gibbs, G. Rupper, C. Ell, O. B. Shchekin, and D. G. Deppe. Vacuum Rabi splitting with a single quantum dot in a photonic crystal nanocavity. Nature, 432:200-203, November 2004.

32. A. Imamoglu et al. Quantum Information Processing Using Quantum Dot Spins and Cavity QED. Physical Review Letters, 83:4204-4207, 1999.

33. L.M. Duan and H.J. Kimble. Scalable Photonic Quantum Computation through Cavity-Assisted Interactions. Physical Review Letters, 92(127902), 2004.

34. L. Childress, J. M. Taylor, A. S. Sorensen, and M. D. Lukin. Fault-tolerant quantum repeaters with minimal physical resources and implementations based on single-photon emitters. Physical Review A, 72(052330), 2005.

35. E. Waks and J. Vučković. Dipole induced transparency in frop-filter cavity-waveguide systems. Physical Review Letters, 80(153601), April 2006.

36. T.D. Ladd, P. van Loock, K. Nemoto K, W.J.Munro WJ, and Y.Yamamoto. Hybrid quantum repeater based on dispersive CQED interactions between matter qubits and bright coherent light. New Journal of Physics, 8:184, 2006.

37. Dirk Englund, Andrei Faraon, Ilya Fushman, Nick Stoltz, Pierre Petroff, and Jelena Vuckovic. Controlling cavity reflectivity with a single quantum dot. Nature, 450(7171), 2007.

38. Y. Akahane, T. Asano, B.-S. Song, and S. Noda. High-Q photonic nanocavity in a two-dimensional photonic crystal . Nature, 425(6961):944-947, October 2003.

39. H. J. Kimble. in Cavity Quantum Electrodynamics, edited by P. Berman. Academic Press, San Diego, 1994.

40. C.J. Hood, M.S. Chapman, T.W. Lynn, and H.J. Kimble. Real-Time Cavity QED with Single Atoms. Physical Review Letters, 80:4157-4160, 1998.

41. A.Auffeves-Garnier, C.Simon, J.M. Gerard, and J.-P. Poizat. Giant optical nonlinearity induced by a single two-level system interacting with a cavity in the Purcell regime. Physical Review A, 75(053823), 2007.

42. G. Nogues, A. Rauschenbeutel, S. Osnaghi, M. Brune, J. M. Raimond, and S. Haroche. Seeing a single photon without destroying it. Nature, 400:239-242, 1999. 